\title{
Mujeres artistas en España en la Edad Moderna: Un mapa a través de la historiografía española
}

\author{
Daniel Lavín González ${ }^{1}$
}

Recibido: 28 de febrero de 2018 / Aceptado: 7 de mayo de 2018

Resumen. A lo largo de la historia, las mujeres han sido marginadas. El mundo del arte no ha sido una excepción. El principal problema al que se enfrentaron las mujeres artistas no fue una cuestión de talento, sino de condición. Todas ellas tuvieron que lidiar con una serie de dificultades que les impidieron desarrollar su talento y habilidades artísticas con libertad. Esta desconsideración parece haberse mantenido en la historiografía durante los siglos posteriores. Durante mucho tiempo, la historia del arte ha ignorado la labor artística de estas mujeres, lo que ha dificultado enormemente el trabajo de los investigadores contemporáneos. Aun así, los estudios sobre mujeres artistas han proliferado poco a poco durante las últimas décadas. Nuestro objetivo con este artículo es realizar un recorrido a través de la historiografía que nos permita conocer la evolución de los estudios realizados en nuestro país sobre las mujeres artistas en la España de la Edad Moderna.

Palabras Clave: Mujeres artistas; Edad Moderna; España; Historiografía.

\section{[en] Women artists in Spain in the Modern Age: A map through the Spanish historiography}

\begin{abstract}
In history, women have been marginalized. The art world has not been an exception. The main problem facing women artists was not a matter of talent, but condition. All of them had to deal with a series of difficulties that prevented them from developing their talent and artistic abilities with freedom. This lack of consideration seems to have remained in historiography for the subsequent centuries. For a long time, the history of art has ignored the artistic work of these women, which has greatly hampered the work of contemporary researchers. Even so, studies on female artists have gradually proliferated over the recent decades. Our objective with this article is to take a journey throughout historiography that allows us to know the evolution of the studies carried out in our country about women artists in the Spain of the Modern Age.
\end{abstract}

Keywords: Women artists; Modern Age; Spain; Historiography.

Sumario. 1. Mujeres artistas en la historiografía española. 2. Primer periodo: Las biografías de la Edad Moderna. 3. Segundo periodo: Los diccionarios biográficos y otras fuentes. 4. Tercer periodo: Estudios de género.

Cómo citar: Lavín González, D. (2018) Mujeres artistas en España en la Edad Moderna: Un mapa a través de la historiografía española, en Anales de Historia del Arte n 28 (2018), 69-85.

\footnotetext{
$1 \quad$ Universidad Complutense de Madrid

dlavin@ucm.es

Código ORCID: 0000-0002-8631-8145
} 


\section{Mujeres artistas en la historiografía española}

Realizar un estado de la cuestión no es un proceso fácil, sin embargo, es completamente imprescindible para llevar a cabo cualquier investigación. Tampoco es sencillo presentarlo a los demás de una forma amena. Enumerar una lista de obras, por orden cronológico y señalando sus principales aportaciones, puede ser un proceso abrumador. Cuando inicié mi investigación sobre mujeres artistas en España durante la Edad Moderna ${ }^{2}$, rápidamente me di cuenta de que exponer un estado de la cuestión sobre este tema sería todavía más complicado. Los pocos datos que se conocen sobre estas mujeres se encuentran muy dispersos en obras y estudios que, en la mayoría de ocasiones, nada tienen que ver con el tema que estamos tratando, al menos no de manera protagonista. La lista de obras que conformarían dicho estado de la cuestión sería demasiado extensa y heterogénea.

Por ello, me ha parecido más acertado estructurar este artículo como un recorrido a través de la historiografía, planteada esta como una especie de mapa bibliográfico donde podemos ir viendo los diferentes tipos de obras y estudios que nos encontramos sobre el tema a lo largo de la historia. Aunque no nos centraremos en todas las obras que pueden existir con referencias a mujeres artistas (sería algo totalmente inabarcable), sí que mencionaremos aquellas que resulten más destacables e interesantes por su aportación dentro del tipo de estudio que estemos tratando en cada momento.

De igual modo que articulamos la historia del arte en periodos para poder construir un relato histórico, este recorrido se encontrará dividido en tres periodos principales. El primero de ellos abarca aquellos textos que fueron escritos en la propia Edad Moderna. Técnicamente, este periodo no formaría parte de la historiografía, pero creemos que la forma en que se presentaba a estas mujeres en su propia época es una buena introducción para entender la historiografía posterior. El segundo periodo nos introduce poco a poco en la historiografía con las primeras obras que podemos encontrar sobre el tema durante el siglo XIX y primera mitad del XX (hasta los años 60 , incluidos). Es dentro del tercer periodo, que abarca desde la segunda mitad del siglo XX hasta nuestros días, donde encontramos la mayor cantidad de bibliografía sobre el tema. Por ello, las obras de este periodo se dividirán en dos categorías principales: las que tratan de mujeres artistas de manera directa y las que lo hacen de una forma indirecta. Con esta división esperamos que el recorrido que estamos a punto de realizar a través de la historiografía española sobre mujeres artistas en España en la Edad Moderna resulte más cómodo y asequible para el lector.

\section{Primer periodo: Las biografías de la Edad Moderna}

El primer periodo en el que hemos dividido la historiografía estaría formado por aquellas obras y textos escritos en vida de las propias mujeres artistas, es decir, de

Esta investigación forma parte de una tesis doctoral que estoy realizando bajo la dirección de Beatriz Blasco Esquivias, enmarcada en el proyecto $\mathrm{I}+\mathrm{D}+\mathrm{i}$ «Femenino singular: Las mujeres y las artes en la corte española en la Edad Moderna (reinas, nobles, artistas y empresarias)», financiado por el Ministerio de Economía y Competitividad y el Fondo Europeo de Desarrollo Regional, HAR2015-65166-P (MINECO/FEDER). 
manera contemporánea a ellas durante la propia Edad Moderna (en vida de ellas o unos años / décadas inmediatamente posteriores).

Durante esta época, la Historia del Arte todavía no existía como una disciplina científica, por lo que todavía no sería correcto hablar de bibliografía, sino de fuentes $^{3}$. Son comentarios coetáneos a estas mujeres, y aunque son la base para muchos de los estudios que se han realizado posteriormente, debemos tomar con cautela algunas de sus referencias ya que no cuentan con el peso de una verificación científica acreditada. Cabe destacar que la mayoría de estas menciones son positivas, halagando casi siempre el trabajo realizado por las mujeres artistas. Sin embargo, no podemos evitar pensar si estos halagos son por el hecho de que una mujer llevara a cabo este tipo de actividades o por la verdadera calidad de sus obras ${ }^{4}$.

Uno de los primeros tratados en los que podemos encontrar referenciadas algunas mujeres artistas es Discursos practicables del nobilísimo arte de la pintura, sus rudimentos, medios y fines que ensena la experiencia con los ejemplares de obras insignes de artifices ilustres, escrito por Jusepe Martínez entre 1673 y $1675^{5}$. El objetivo principal de esta obra, como su propio nombre indica, es enseñar aquellos principios necesarios para llevar a cabo el arte de la pintura de la forma más acertada posible. Entre estas lecciones también encontramos la mención de algunos artistas destacables de su momento, incluidas varias mujeres que llevaban a cabo esta actividad. Algunas de las mujeres mencionadas son las pintoras Ana Lastanosa ${ }^{6}$, Teresa Huesca (también destacó por sus grabados) ${ }^{7}$, la hija de Felipe Liaño ${ }^{8}$ o Dionisia de Segura, que realizó dibujos y cuadros bordados ${ }^{9}$.

En la obra de José García Hidalgo, Principios para estudiar el nobilísimo y real arte de la pintura, escrita en $1693^{10}$, se hace mención a cuatro mujeres artistas en el prólogo: Sofonisba Anguissola, Teresa Sarmiento de la Cerda (mencionada como Duquesa de Béjar), María de Guadalupe y María Abarca ${ }^{11}$, todas ellas pintoras. El propio autor dice conocer a más, pero se escusa por no mencionarlas ya que, según él, no quiere extenderse demasiado.

3 Aunque este primer periodo no forma parte de la historiografía española como tal, nos ha parecido interesante incluir y mencionar las obras coetáneas a dichas mujeres donde encontramos las primeras referencias. Este primer periodo puede ser visto como una especie de prólogo para todas las obras que mencionaremos posteriormente.

$4 \quad$ No queremos restar mérito al trabajo realizado por estas mujeres artistas, pero no podemos evitar tener esta sensación cuando leemos algunas de las referencias dejadas por estos autores. Casi nunca se mencionan que obras realizó u otros datos que en el caso de artistas masculinos siempre aparecen, como por ejemplo, su propio nombre.

5 Destacamos la edición publicada por la Real Academia de Bellas Artes de San Fernando en 1866 porque incluye numerosas notas que complementa la información dada por el autor original. En algunos casos, esta información nueva es sobre algunas mujeres artistas. Martínez, J. (1866). Discursos practicables del nobilísimo arte de la pintura, sus rudimentos, medios y fines que ensena la experiencia con los ejemplares de obras insignes de artifices ilustres ( $1^{\circ}$ ed. Zaragoza, 1673/75). Madrid: Academia de Bellas Artes de San Fernando.

Ibídem, 33.

Ibidem.

Ibidem, 127 y 128.

Ibidem, 34.

10 Podemos encontrar muchas ediciones sobre la obra de José García Hidalgo, sin embargo, destacamos la de 2006 por ser una edición crítica e incluir un segundo tomo con varios estudios sobre la obra (ninguno de ellos es sobre las pocas mujeres artistas que menciona, pero complementan muy bien el contenido del texto original). García Hidalgo, J. (2006). Principios para estudiar el nobilísimo y real arte de la pintura, Tomo I y II. Valencia: Universidad Politécnica de Valencia, Terátos.

$11 \quad$ Ibidem, 3. 
En Varia historia de sanctas e ilustres mujeres en todo genero de virtudes, publicada en 1583 y dividida en tres libros, Juan Pérez de Moya nos hace un repaso por una gran cantidad de mujeres destacables a lo largo de la historia ${ }^{12}$. El primer libro está dedicado a mujeres santas, el segundo a heroicas en la guerra o el gobierno y el tercero a las doctas en varias ciencias y artes. Es en este tercer libro, concretamente en el capítulo LVI, donde el autor habla de las mujeres que se dedicaron a la pintura. Entre ellas menciona a las pintoras Isabel de Varros ${ }^{13}$, María de Jesús ${ }^{14}$ e Isabel Sánchez Coello, a la que describe de manera notable sobre las demás y la presenta como una mujer muy virtuosa en diferentes áreas ${ }^{15}$.

Uno de los autores más destacables de esta época es Antonio Palomino de Castro y Velasco con su obra El museo pictórico y la escala óptica, dividida en tres volúmenes. En el tercero, El Parnaso pintoresco laureado (publicado junto al segundo en 1724), el autor nos presenta la biografía de varios artistas importantes de su época. Entre ellas podemos encontrar a varias mujeres artistas. Aunque solo tres cuentan con su propia biografía (Luisa Roldán ${ }^{16}$, Sofonisba Anguissola ${ }^{17}$ y una tal Sofonisba Gentilesca ${ }^{18}$ ), podemos encontrar muchas más integradas en las biografías de otros artistas masculinos. Eran hijas, mujeres o hermanas de dicho artista y el autor las menciona de forma más o menos extensa cuando habla de él. Algunos ejemplos son Magdalena Gilarte ${ }^{19}$, Isabel Carrasquilla ${ }^{20}$ o Jesualda Sanchís ${ }^{21}$. Por desgracia, en la mayoría de los casos, apenas se aporta información más allá de su existencia y la relación que tenían con dicho artista. También encontramos algunas referencias en el primer tomo de la obra, Theórica de la pintura (publicado en 1715), cuando el autor hace un repaso por varias mujeres artistas de la historia y profundiza un poco en aquellas españolas pertenecientes a la nobleza que llevaban a cabo este tipo de acti-

12 Podemos destacar la edición que se encuentra incluida dentro de la obra Juan Pérez de Moya: Obras completas, dividida en dos volúmenes y editada por Consolación Baranda. En el primer volumen de la obra se incluyen las obras «Comparaciones o símiles para los vicios y virtudes» $\mathrm{y}$ «Philosophía secreta», mientras que en el segundo encontramos «Arithmética práctica y speculativa» y «Varia historia de sanctas e illustres mugeres». Pérez de Moya, J. (1998). Varia historia de sanctas e ilustres mujeres en todo genero de virtudes. En C. Baranda (ed.), Juan Pérez de Moya: Obras completas (pp. 613-1004). Madrid: Fundación José Antonio de Castro.

13 Ibidem, 999.

14 Ibidem.

15 Ibidem.

16 Palomino de Castro y Velasco, A. (1796, $1^{\circ}$ ed. 1724). El museo pictórico y la escala óptica III: El Parnaso español pintoresco laureado. Madrid: Imprenta de Sancha, 684-686.

17 Ibidem, 373-375.

18 Ibídem, 386; Esta referencia hecha por el autor es errónea. El nombre de Sofonisba Gentilesca es resultado de mezclar los nombres de Sofonisba Anguissola y Artemisia Gentileschi. El origen de este error lo encontramos en la obra de Lázaro Díaz del Valle y de la Puerta, Origen y Yllustracion del Nobilisimo y Real Arte de la Pintura y Dibuxo, escrita en 1659, donde el autor habla de algunas mujeres artistas como Lavinia Fontana, Magdalena de Van de Paz, Artemisia Gentileschi y la mencionada Sofonisba Gentilesca, que en realidad es Sofonisba Anguissola pero el autor confunde los apellidos con la anterior. Las fuentes de Lázaro Díaz del Valle son Pacheco y Butrón, donde también se producen varias equivocaciones sobre esta mujer. Cuando Antonio Palomino tomó algunas referencias de esta obra para su tratado, como hicieron otros autores posteriores, incluyó este error. Sobre la obra de Lázaro Díaz del Valle destacamos el estudio de David García López, donde se incluye una versión transcrita del tratado con una gran cantidad de anotaciones e información complementaria. También se profundiza en este error. García López, D. (2008). Lázaro Díaz del Valle y las Vidas de pintores de España. Madrid: Fundación Universitaria Española.

19 Ibídem, 678.

20 Ibidem, 644.

21 Ibídem, 731. 
vidades para así justificar la propia nobleza de la pintura. Es destacable que incluya, aunque sea muy brevemente, a su hermana entre estas mujeres ${ }^{22}$.

Algo más tardía es la obra Diccionario histórico de los más ilustres profesores de bellas artes en España, escrita por Juan Antonio Ceán Bermúdez en 1800. Con un planteamiento muy similar al tomo tercero de Palomino, nos encontramos ante un diccionario en el que se recopila la biografía de varios artistas del momento y hay varias mujeres artistas entre ellos. De hecho, el número de referencias es superior al de autores anteriores, ya que muchas de estas mujeres cuentan con su propia referencia biográfica. Desde la pintora María de $\mathrm{Abarca}^{23}$, que inicia el diccionario, hasta María Ignacia y Rosalía Navarro ${ }^{24}$, pintoras que aprendieron el dibujo de su padre el Marqués de Victoria, el número de mujeres que podemos encontrar a lo largo de los seis tomos que componen la obra es realmente destacable.

Aunque son excepciones, dentro de este primer periodo también existen algunos textos escritos por las propias mujeres, todos ellos con un carácter autobiográfico. Uno de los más destacables es el texto escrito por Estefanía de la Encarnación, una monja franciscana en el monasterio de Santa Clara en Lerma que escribió su propia autobiografía ${ }^{25}$. Este tipo de obras nos permiten conocer más destalles sobre la vida y producción de algunas mujeres concretas, pero también nos aportan datos que son aplicables a todas las mujeres de la época. En este mismo texto, por ejemplo, Estefanía nos hace mención de Beatriz Villena, otra mujer que realizó estas actividades y a la que ella mismo enseñó dibujo ${ }^{26}$.

\section{Segundo periodo: Los diccionarios biográficos y otras fuentes}

Tras este primer periodo, las menciones sobre mujeres artistas españolas en la Edad Moderna siguen siendo algo prácticamente anecdótico en la historiografía. Podemos encontrar algunas referencias sobre mujeres de fama, pero son excepciones en comparación con la mayoría. En vida, estas mujeres eran halagadas y mencionadas en varias obras, sin embargo, tras su muerte, como si hubieran sido una moda pasajera, casi ningún autor se acuerda de ellas.

No será hasta el segundo periodo en el que hemos dividido la historiografía, que abarcaría el siglo XIX y primera mitad el XX (hasta los años 60), cuando el tema empieza a cobrar importancia poco a poco.

Durante el siglo XIX podemos encontrar varios diccionarios en los que aparecen algunas mujeres artistas. El primero del que debemos hacer mención es Diccionario Biográfico Universal de Mujeres Célebres, escrito por Vicente Díez Canseco en 1844. Formado por tres tomos, este diccionario intenta recopilar a todas las mujeres importantes de la historia. Desde aquellas que aparecen en el Antiguo Testamento

22 Palomino de Castro y Velasco, A. (1795, $1^{\circ}$ ed. 1715). El museo pictórico y la escala óptica I: Theórica de la pintura. Madrid: Imprenta de Sancha, 188.

23 Ceán Bermúdez, J.A. (1800). Diccionario histórico de los más ilustres profesores de las bellas artes en España, Tomo I: A-C. Madrid: Imprenta de la viuda de Ibarra, 1.

24 Céan Bermúdez, J.A. (1800). Diccionario histórico de los más ilustres profesores de las bellas artes en España, Tomo VI: X-Z. Madrid: Imprenta de la viuda de Ibarra, 90.

25 Estefanía de la Encarnación (O.S.C). La vida de Soror Estephania de la Encarnaçion, Monja Proffessa en el Monasterio de Religiossas Françiscas de Nuestra Madre Santa Clara, en esta Villa de Lerma, año de XDCXXI, [escrita por ella misma, manuscrito del siglo XVII]

26 Ibídem, 28. 
hasta las reinas de los años más recientes. Como podemos imaginar, también aparecen algunas artistas. Entre las españolas mencionadas encontramos, por ejemplo, a las grabadoras María Eugenia de Beer $^{27}$ y Ana Heylan ${ }^{28}$ o las pintoras Mariana Cuevas Benavides ${ }^{29}$ y María de Loreto Prieto ${ }^{30}$. Algunas de ellas, como Bárbara María de Hueba ${ }^{31}$ o Ángela Pérez Caballero ${ }^{32}$, ambas pintoras, ya fueron citadas por autores anteriores, como Ceán Bermúdez ${ }^{33}$.

También debemos destacar Adiciones al diccionario histórico de los más ilustres profesores de las bellas artes en España de D. Juan Agustín Ceán Bermúdez, escrito por Cipriano Muñoz y Manzano, conde de Viñaza ${ }^{34}$, que funciona como complemento sobre la obra original de Ceán Bermúdez. En ella podemos encontrar desde referencias a artistas completamente nuevas hasta información complementaria de algunos que ya aparecían en el diccionario original. Entre ellas, también aparecen varias mujeres artistas, como la platera María Benavente ${ }^{35}$, la pintora Micaela Fe$\operatorname{rrer}^{36}$ o la escultora Soror Mencía de la Olivia ${ }^{37}$. El propio autor dice que gran parte de estas adiciones se corresponden a Valentín Carderera y Solano ${ }^{38}$.

En el Diccionario biográfico de artistas valencianos de José Ruiz de Lihory y Pardines también hay algunas referencias a mujeres pintoras, como Margarita y Dorotea Joanes ${ }^{39}$ o Josefa María Larraga ${ }^{40}$. No aparece ninguna mencionada en los apartados de escultura y arquitectura que incluye el diccionario.

Es durante la primera mitad del siglo XX, hasta los años 60, cuando empezamos a encontrar obras que contienen un gran número de referencias sobre mujeres artistas españolas de la Edad Moderna. Dos de los ejemplos más destacables son Las pintoras españolas: boceto histórico-biográfico, de José Parada y Santín (1902) ${ }^{41}$, y Galería Universal de pintoras, de Carmen Pérez-Neu $(1964)^{42}$. Sin embargo, ambas

27 Díez Canseco, V. (1844). Diccionario Biográfico Universal de Mujeres Célebres, Tomo I. Madrid: Imprenta de D. José Félix Palacios, 272.

28 Díez Canseco, V. (1844). Diccionario Biográfico Universal de Mujeres Célebres, Tomo II. Madrid: Imprenta de D. José Félix Palacios, 236.

29 Díez Canseco, op. cit., Tomo I, 1844, 599.

30 Díez Canseco, V. (1845). Diccionario Biográfico Universal de Mujeres Célebres, Tomo III. Madrid: Imprenta de D. José Félix Palacios, 331.

31 Díez Canseco, op. cit., Tomo II, 1844, 270.

32 Díez Canseco, op. cit., Tomo III, 1845, 294.

33 En estos casos, el autor casi siempre lo menciona y transcribe lo dicho en este diccionario para así complementar el comentario.

34 La obra se encuentra dividida en cuatro volúmenes. El primero de ellos abarca la Edad Media y los tres siguientes se centran en los siglos XVI, XVII y XVIII. Los dos primeros volúmenes fueron publicados en 1889 y los dos siguientes en 1894.

35 Muñoz y Manzano, C. (1889). Adiciones al diccionario histórico de los más ilustres profesores de las bellas artes en España de D. Juan Agustín Ceán Bermúdez, Tomo II. Madrid: Tipografía de los Huérfanos, 55.

36 Ibidem, 194.

37 Muñoz y Manzano, C. (1894). Adiciones al diccionario histórico de los más ilustres profesores de las bellas artes en España de D. Juan Agustín Ceán Bermúdez, Tomo III. Madrid: Tipografía de los Huérfanos, 194-195.

38 En la Real Academia de Bellas Artes de San Fernando se conserva una copia del Diccionario de Ceán Bermúdez que perteneció a Valentín Carderera, llena de anotaciones y correcciones en los márgenes. Esta copia es la base principal de esta obra.

39 Ruiz de Lihory y Pardines, J. (1897). Diccionario biográfico de artistas valencianos. Valencia: Imprenta de Federico Domenech, 178.

40 Ibídem, 182.

41 Parada y Santín, J. (1902). Las pintoras españolas: boceto histórico-biográfico. Madrid: Impr. Del Asilo de Huérfanos del S.C. de Jesús.

42 Pérez-Neu, Carmen G. (1964). Galería universal de pintoras. Madrid: Editora Nacional. 
obras cuentan con el pequeño inconveniente de que, en ciertas ocasiones, no citan la fuente de la que han obtenido la información. Esto ha llevado a que, décadas después, algunos autores hayan dudado sobre algunas de estas referencias o incluso cuestionado la veracidad de ellas.

Pongamos un ejemplo: en su obra, José Parada y Santín habla de María Blanca Ribera, hija de José de Ribera, a la que describe como sobresaliente pintora y señala que fue modelo para algunas obras de su padre ${ }^{43}$. También dice que vivió un romance con el hijo bastardo de Felipe IV, Juan de Austria. Sin embargo, si nos adelantamos un momento en el futuro, Pilar Muñoz López nos muestra en su estudio, tras una profunda investigación sobre el artista, que José de Ribera no tuvo ninguna hija cuyo nombre fuera María Blanca. Lo más próximo es una sobrina llamada María Rosa, que si tuvo un romance con Juan de Austria pero nada nos indica que ejerciera como pintora. Esto mismo ocurre con los demás hijos e hijas del artista ${ }^{44}$. Además, ningún otro autor, de la época o posterior, hacen mención de esta hipotética hija.

Con esto no queremos decir que el autor se inventara esta referencia o que toda la obra esté equivocada. Lo más probable es que Parada y Santín cruzara datos y se equivocara, pero como no cita la fuente de la que ha obtenido la información, no podemos saber dónde se ha producido el error. De hecho, nos encontramos ante una obra muy destacable con una gran cantidad de referencias, casi todas ellas acertadas. Si solo hablamos de las mujeres artistas españolas de la Edad Moderna, encontramos más de 60 mujeres mencionadas (de otros países y periodos se menciona muchas más). Sin embargo, de aquellas que no cita la fuente, nos vemos en la obligación de contrastarlas antes de darlas por válidas.

Otra mención que podría resultar polémica, por así decirlo, es la que hace este mismo autor de Juana Pacheco, hija de Francisco Pacheco y mujer de Diego Velázquez. José Parada y Santín afirma que, aunque no se conservan obras suyas (al menos conocidas), esta mujer debió ser muy notable en el arte de la pintura al estar rodeada de artistas tan importantes ${ }^{45}$. Se apoya en el cuadro conocido como Sibila, de Diego Velázquez, donde se ve a una mujer con una tablilla a punto de empezar a realizar una obra. El autor dice que esta mujer es igual a la figura de Juana Pacheco que aparece en la obra de Juan Bautista Martínez de Mazo, La familia del pintor, donde se representa a toda su familia. Sin embargo, no tenemos constancia de que esta mujer llevara a cabo ningún tipo de producción artística. Ni su padre ni otros autores de la época se refieren a ella como artista. Este ejemplo no sería como el de María Blanca Ribera pues el propio autor nos dice que otros investigadores no apoyan esta hipótesis, incluso menciona a varios compañeros y cuáles son las ideas contrarias que dan. Él mismo admite que realmente no tiene ninguna prueba pictórica, más allá de los ejemplos mencionados anteriormente, que lo demuestre.

Algo similar ocurre con la obra de Carmen Pérez-Neu que hemos mencionado anteriormente. No obstante, no podemos dejar de recalcar la importancia y mérito que tienen estas obras. Pese a algún inconveniente, el número de referencias e información que podemos hallar sobre algunas mujeres artistas que se encontraban en España en la Edad Moderna es realmente destacable.

\footnotetext{
43 Parada y Santín, op. cit., 1902, 28.

44 Muñoz López, P. (2003). Mujeres españolas en las artes plásticas: pintura y escultura. Madrid: Síntesis, 69-71.

45 Parada y Santín, op. cit., 1902, 26.
} 
Durante estos años, también surgen estudios que se especializan en mujeres concretas. Un ejemplo destacable sería Dos monjas vallisoletanas poetisas, por Blanca Alonso-Cortés, donde se trata la figura de Cecilia Sobrino, conocida como Cecilia del Nacimiento cuando tomó los hábitos, y su madre, Cecilia Morillas ${ }^{46}$.

Como hemos visto durante el siglo XIX, y a lo largo de la historiografía, también podemos encontrar obras donde aparecen mencionadas mujeres artistas de manera más o menos colateral. Por ejemplo, en la obra de José Gestoso y Pérez, Biografía del pintor sevillano Juan de Valdés Leal, se hace mención de Isabel Carrasquilla, María y Luisa Valdés, la mujer e hijas del pintor que protagoniza la biografía ${ }^{47}$. Lo mismo ocurre en Juan de Juanes: una familia de pintores, de Ángel Dotor y Municio, donde aparecen mencionadas y tratadas las dos hijas de Juan de Juanes, Dorotea y Margarita Macip (ya las hemos mencionado anteriormente con el apellido Joanes) ${ }^{48}$. En el artículo de Martín S. Soria, «Notas sobre algunos bodegones españoles del siglo XVII», se hace especial mención a la producción artística de Josefa de Ayala ${ }^{49}$. Sobre esta misma mujer, encontramos la obra Josefa de Ayala: Pintora Ibérica del siglo $X V I I$, escrita por José Hernández Díaz ${ }^{50}$. Como veremos más adelante, esta es una de las pocas mujeres que ha contado con diversos estudios totalmente dedicados a ella.

\section{Tercer periodo: Estudios de género}

Desde principios del siglo XX, la historiografía española sobre mujeres artistas en España durante la Edad Moderna ha ido aumentando de manera progresiva hasta nuestros días. Sin embargo, es durante las últimas décadas (a partir de los años 70 , más o menos), cuando se produce un notable impulso. La cantidad de obras y estudios que podemos encontrar sobre el tema durante estos años es realmente destacable, más todavía si lo comparamos con los siglos anteriores. Para que no resulte tan confuso abordar este tercer periodo en el que hemos dividido la historiografía, lo mejor será mostrarlo a su vez en dos categorías principales:

- En primer lugar, tendríamos aquellas obras que se centran en las mujeres artistas y proyectan una perspectiva de género en su estudio sobre ellas. Encontramos muy pocas que estén centradas exclusivamente en la Edad Moderna pues la mayoría trata artistas contemporáneas. A partir de los años 70 se produce un cambio, ya no se trata únicamente de recuperar una lista de nombres o realizar biografías de artistas. En esto, Estrella de Diego es una de las autoras más importantes y representativas, pionera en la incorporación de los estudios de género en España. Aunque su tesis está centrada en las mujeres del siglo XIX y primeros años del XX, como su propio nombre indica, La mujer y la pintura en la España del siglo XIX. Mujeres pintoras en Madrid: 1868-1910, en ella dedica una parte importante a las mujeres de periodos

46 Alonso-Cortés, B. (1944). Dos monjas vallisoletanas poetisas. Valladolid: Imprenta Castellana.

47 Gestoso y Pérez, J. (1917). Biografía del pintor sevillano Juan de Valdés Leal. Sevilla: Oficina Tipográfica de Juan P. Gironés.

48 Dotor y Municio, Á. (1953). Juan de Juanes: una familia de pintores. Gerona: Dalmáu Carles Pla.

49 Soria, Marín S. (1959). Notas sobre algunos bodegones españoles del siglo XVII. Archivo Español de Arte, 128, 273-280.

50 Hernandez Díaz, J. (1967). Josefa Ayala: Pintora Ibérica del siglo XVII. Sevilla: Ayuntamiento de Sevilla, Delegación de Cultura. 
anteriores (desde la Edad Media, incluyendo a varias de la Edad Moderna) ${ }^{51}$. La tesis fue presentada en 1986 bajo la dirección de Antonio Bonet Correa en la Universidad Complutense de Madrid y se publicó revisada en 2009 por la editorial Cátedra, con el título La mujer y la pintura del XIX español: cuatrocientas olvidadas y alguna más ${ }^{52}$. No es casualidad que Estrella de Diego sea pionera de estos estudios en nuestro país, pues participó en alguno de los seminarios organizados por la propia Linda Nochlin durante los años 70 y vivió de cerca el origen de la teoría feminista en la historia del arte.

En la obra de Rosa Elvira Caamaño Fernández, Educación y dedicación: Aportaciones de las grandes pintoras al arte desde la Antigüedad hasta 1.800, la autora hace un repaso por la historia, hablando de todos los tipos de mujeres artistas que podemos encontrar en el mundo del arte (pintoras, escultoras, bordadoras, miniaturistas, etc.), con algunos ejemplos representativos ${ }^{53}$. Uno de los capítulos del libro se encuentra dedicado a las mujeres artistas españolas de la Edad Moderna ${ }^{54}$.

Un estudio importante sobre mujeres artistas es la aportación de Patricia Mayayo, Historias de mujeres, historias del arte, en $2003^{55}$. Aunque esta obra no profundiza en las mujeres artistas de la Edad Moderna, sí que presenta un notable estado de la cuestión sobre las mujeres artistas en general y la metodología apropiada para los estudios de género. Se citan numerosos ejemplos de mujeres y las obras que realizaron, pero también se aborda de manera importante el estudio de estas y la forma en que se ha desarrollado durante las últimas décadas, citando numerosos autores y notable bibliografía sobre el tema.

Otros autores, como Álvaro Molina, han profundizado de manera muy destacable en los estudios de género durante estos últimos años ${ }^{56}$. María Elena Díez Jorge, investigadora especializada en el marco histórico del mudéjar, tiene numerosos libros y artículos sobre la mujer, no siempre como artista, y los estudios de género dentro de este ámbito ${ }^{57}$. También podemos destacar el artículo de Marta Mantecón Moreno para la revista Anales de Historia del Arte, "“Tú tampoco tienes nada": arte feminista y de género en la España de los 90», donde se nos presenta la evolución del género y el arte feminista en nuestro país durante los años $90^{58}$.

51 Diego Otero, E. de (1987). La mujer y la pintura en la España del siglo XIX. Mujeres pintoras en Madrid: 1868 1910, Tomo I y II. Madrid: Universidad Complutense de Madrid, Facultad de Geografía e Historia.

52 Diego Otero, E. de (2009). La mujer y la pintura del XIX español: cuatrocientas olvidadas y alguna más. Madrid: Cátedra.

53 Caamaño Fernández, R. E. (2001). Educación y dedicación: Aportaciones de las grandes pintoras al arte desde la Antigüedad hasta 1.800. Vigo: Servizo de Publicacions de Universidade de Vigo.

54 El capítulo se titula «Artistas españolas» (pp. 116-130) y como ocurre en otros capítulos del libro, la estructura de este nos muestra el contexto en que se encontraban dichas mujeres y nos menciona algunos ejemplos representativos.

55 Mayayo, P. (2003). Historias de mujeres: historias del arte. Madrid: Cátedra.

56 Álvaro Molina ha tratado de manera muy extensa los estudios de género en la España ilustrada del siglo XVIII en algunas de sus obras. Destacamos Molina, A. (2012). Visualizando el género en la Historia del Arte. El siglo XVIII español como caso de estudio. Anuario del Departamento de Historia y Teoría del Arte, 24, 79-92; Molina, A. (2013). Mujeres y hombres en la España ilustrada: Identidad, género y visualidad. Madrid: Cátedra.

57 De entre todas las obras y colaboraciones de María Elena Díez Jorge, podríamos señalar los siguientes estudios de género y las mujeres: Díez Jorge, M. E. (ed.) y Sánchez Romero, M. (ed.) (2010). Género y paz. Madrid: Icaria; Díez Jorge, M. E. (2011). Mujeres y arquitectura: mudéjares y cristianas en la construcción. Granada: Editorial Universidad de Granada; Díez Jorge, M. E. (ed.) (2015). Arquitectura y mujeres en la historia. Madrid: Síntesis.

58 Mantecón Moreno, M. (2010). "Tú tampoco tienes nada": arte feminista y de género en la España de los 90. Anales de Historia del Arte, Volumen extraordinario, 155-167. 
En la obra Mujeres españolas en las artes plásticas: pintura y escultura, Pilar Muñoz López hace un repaso realmente notable por algunas de las mujeres artistas españolas más destacables de las que se tienen constancia en ese momento ${ }^{59}$. Aunque el capítulo 6, «Las mujeres artistas en la época moderna: Renacimiento y Barroco», trata aspectos muy importantes de este periodo, es en el capítulo 7, «Algunas mujeres en la actividad artística del Siglo de Oro», donde se hace mención de varias mujeres artistas españolas de esta época. Otro artículo suyo, de 2009, «Mujeres españolas en las artes plásticas», es un buen complemento sobre lo que ella misma presentó en la obra original y, en términos generales, resulta más concreto en relación con el periodo que nos interesa ${ }^{60}$.

A raíz de su tesis doctoral ${ }^{61}$, Vicent Ibiza i Osca llevó a cabo Obra de mujeres artistas en los museos españoles, guía de pintoras y escultoras: 1500-1936, publicada en $2006^{62}$, y donde encontramos un buen estado de la cuestión sobre las mujeres artistas españolas (desde la Edad Moderna hasta nuestros días), una buena exposición del contexto en el que se encontraban (tanto nacional como internacional) y algunas referencias muy destacables, pues recupera, por ejemplo, la idea que hemos visto antes sobre Juana Pacheco como pintora ${ }^{63}$.

La tesis doctoral de Matilde Torres López, La mujer en la docencia y la práctica artística en Andalucía durante el siglo XIX, realizada bajo la dirección de Rosario Camacho Martínez en la Universidad de Málaga en el año $2007^{64}$, se centra en un área concreta y un periodo más contemporáneo, aunque también trata a las mujeres artistas de la Edad Moderna en sus primeros apartados, a modo de introducción para la parte protagonista del trabajo ${ }^{65}$.

Dentro de esta primera categoría, habría que destacar también la realización de varios eventos dedicados al tema y cuyas actas han sido publicadas. Con motivo del Año Internacional de la Mujer, Matilde López Serrano realizó una conferencia titulada Presencia femenina en las artes del libro español el 19 de noviembre de 1975 en la Fundación Universitaria Española ${ }^{66}$. En ella encontramos referencias a muchas mujeres artistas, desde la ocupación musulmana en la Península hasta el siglo XX. De la Edad Moderna se mencionan varias, como la iluminadora conocida como Monja de Santa Paula ${ }^{67}$, las

59 Muñoz López, op. cit., 2003.

${ }_{60}$ Muñoz López, P. (2009). Mujeres españolas en las artes plásticas. Arte, individuo y sociedad, 21, 73-88.

${ }_{61}$ La tesis se titula Dona i Art a Espanya: Artistes d'abans de 1936. Obra Exposada-Obra Desapareguda y fue realizada en 2004 bajo la dirección de María Teresa Beguiristain Alcorta.

62 Ibiza i Osca, V. (2006). Obra de mujeres artistas en los museos españoles, guía de pintoras y escultoras: 15001936. Valencia: Centro Francisco Tomás y Valiente, UNED Alzira-Valencia.

63 La mayoría de ellas se encuentran en un capítulo titulado «Artistas españolas antes del siglo XVIII» (pp. 83-93), aunque también podemos encontrar algunas más en el capítulo siguiente de la obra, «Arte y artistas españolas de los siglos XVIII y XIX» (pp. 95-111), donde se hace especial mención de aquellas que formaron parte de las Academias de Bellas Artes.

${ }_{64}$ Torres López, M. (2007). La mujer en la docencia y la práctica artística en Andalucía durante el siglo XIX (Tesis Doctoral). Universidad de Málaga, Málaga.

65 Hasta, más o menos, la página 88, la tesis trata a las mujeres artistas que podemos encontrar en España durante la Edad Moderna (contexto, referencias, obras conocidas, etc.). Esta primera parte es una buena introducción para las mujeres artistas en Andalucía durante el siglo XIX, sin embargo, está tan desarrollada que es completamente digna de mención en este recorrido que estamos haciendo a través de la historiografía.

${ }_{66}$ Esta conferencia ha sido publicada en un librito de 38 hojas y 32 ilustraciones por la propia Fundación Universitaria Española. López Serrano, M. (1976). Presencia femenina en las artes del libro español. Madrid: Fundación Universitaria Española.

67 Ibidem, 21. 
grabadoras Ana Heylan ${ }^{68}$, María Eugenia de Beer ${ }^{69}$, Teresa Agüesca $^{70}$, Luisa Morales ${ }^{71}$ y María Monforte $^{72}$, o más recientes, las pintoras Ana Menéndez ${ }^{73}$ y Ana María Mengs ${ }^{74}$.

De todos modos, el primer evento completamente dedicado a las mujeres artistas sería el seminario organizado en 1984 por María Ángeles Durán en la Universidad Autónoma de Madrid, La imagen y la mujer en el arte español, un seminario considerado como uno de los eventos pioneros sobre el tema en nuestro país ${ }^{75}$. Respecto al tema que nos ocupa, cabe destacar el estudio de Alonso E. Pérez Sánchez sobre «Las mujeres "pintoras" en España» ${ }^{76}$. El autor propone una clasificación de mujeres artistas que resulta muy útil para contextualizar el tema en la Edad Moderna: hijas o familiares de artistas, monjas pintoras y pintoras burguesas ${ }^{77}$.

Del 26 al 29 de noviembre de 1996 se celebraron las VIII Jornadas de Arte en el Centro de Estudios Históricos, dirigidas por Wifredo Rincón García y con el título de La mujer en el arte españo $l^{78}$. Aunque el contenido de dichas jornadas aborda la figura de la mujer a lo largo de toda la historia del arte, no solo como artista sino también como imagen representada, encontramos un número muy destacable de ponencias dedicadas a las mujeres artistas en España durante la Edad Moderna: «La mujer y el retrato cortesano del siglo XVI visto a través de la obra de Sofonisba Anguissola, maestra de pintura y dama de honor de Isabel de Valois», de María Kusche ${ }^{79}$; «La mujer y el arte en los documentos de los siglos XVI y XVII: Los miembros femeninos de la familia de los Leoni», de Magarita Estella Marcos ${ }^{80}$; «La presencia de la mujer en los oficios artísticos», de Guadalupe Ramos de Castro ${ }^{81}$; «La mujer en el arte madrileño del siglo XVII», de José Manuel Cruz Valdovinos ${ }^{82}$; «Isabel de Farnesio, la pasión por el arte», de Teresa Lavalle $\mathrm{Cobo}^{83}$; «Reconsiderando el papel de la mujer en la Real Academia de Bellas Artes de San Fernando», de Theresa

\footnotetext{
Ibidem, 22-24.

Ibidem, 24-26.

Ibidem, 26.

Ibidem, 26-27.

Ibidem, 28.

Ibidem.
}

74 Ibídem, 28-29.

75 Durán, M. A. (dir.) (1984). La imagen de la mujer en el arte español. Madrid: Universidad Autónoma de Madrid.

76 Pérez Sánchez, A. E. (1984). Las mujeres “pintoras” en España. En M. A. Durán (dir.), La imagen de la mujer en el arte español (pp. 73-102). Madrid: Universidad Autónoma de Madrid.

77 Algunas mujeres podrían estar incluidas en, al menos, dos de las categorías. Muchas hijas de artistas o mujeres pertenecientes a la nobleza que practicaban este arte acababan entrando en religión y continuaban con sus prácticas en este nuevo contexto.

78 Rincón Garcia, W. (dir.) (1997). La mujer en el arte español: Actas de las VIII Jornadas de Arte. Madrid: Alpuerto.

79 Kusche, M. (1997). La mujer y el retrato cortesano del siglo XVI visto a través de la obra de Sofonisba Anguissola, maestra de pintura y dama de honor de Isabel de Valois. En W. Rincón García (dir.), La mujer en el arte español: Actas de las VIII Jornadas de Arte (pp. 69-80). Madrid: Alpuerto.

80 Estella Marcos, M. (1997). La mujer y el arte en los documentos de los siglos XVI y XVII: Los miembros femeninos de la familia de los Leoni. En W. Rincón García (dir.), La mujer en el arte español: Actas VIII Jornadas de Arte (pp. 117-130). Madrid: Alpuerto.

81 Ramos de Castro, G. (1997). La presencia de la mujer en los oficios artísticos. En W. Rincón García (dir.), La mujer en el arte español: Actas de las VIII Jornadas de Arte (pp. 169-178). Madrid: Alpuerto.

82 Cruz Valdovinos, J. M. (1997). La mujer en el arte madrileño del siglo XVII. En W. Rincón García (dir.), La mujer en el arte español: Actas de las VIII Jornadas de Arte (pp. 179-186). Madrid: Alpuerto.

83 Lavalle Cobo, T. (1997). Isabel de Farnesio, la pasión por el arte. En W. Rincón García (dir.), La mujer en el arte español: Acatas de las VIII Jornadas de Arte (pp. 217-228). Madrid: Alpuerto. 
Ann Smith ${ }^{84}$; «Las escuelas femeninas de encajes en el Madrid ilustrado», de Ángel López Castán ${ }^{85}$; y «La mujer en la creación artística del barroco canario», de Carlos Javier Castro Brunetto ${ }^{86}$. Podríamos mencionar otras ponencias sobre mujeres mecenas o coleccionistas de esta misma época dentro de estas Jornadas, sin embargo, solo hemos destacado las intervenciones dedicadas directamente a las mujeres artistas.

Hay otros artículos de revistas o capítulos de libros dedicados al tema. Elena López Palomares escribió «Mujeres en la Real Academia de Bellas Artes de San Carlos: 1768 - 1849» para la revista Asparkía: Investigació Feminista en 1995, donde analiza los casos de varias mujeres artistas que se encontraron en la Real Academia de Bellas Artes de San Carlos de Valencia, como las pintoras María Caro y Sureda, Micaela Ferrer, o Engracia de las Casas y Manuela Mercader y Caro, que llegaron a ser nombradas Directoras Honorarias de la institución ${ }^{87}$.

Dentro de la obra Roldana, dirigida por José Luis Romero Torres y Antonio Torrejón Díaz con motivo de la exposición celebrada en el Real Alcázar de Sevilla del 25 de julio al 14 de octubre de 2007, se incluye un capítulo titulado «Ser mujer y artista en la España de la Edad Moderna», escrito por Ana Aranda Bernal ${ }^{88}$. Aunque Luisa Roldán es el eje central del texto, la autora aprovecha esto para desarrollar el contexto general en el que se encontraban las artistas de esta época y hablar de muchos más ejemplos. Algunos de ellos son realmente interesantes ya que no se encuentran en casi ningún otro estudio, como el de María Gutiérrez de Valdelomar, una mujer a la que no querían examinar por su condición femenina y de la que tenemos sus cartas de protesta en el Archivo General de Simancas ${ }^{89}$.

Dentro de esta primera categoría encontramos otras monografías dedicadas a mujeres artistas de cuya vida y producción conservamos más información y han generado más estudios especializados ${ }^{90}$, aunque siguen siendo excepcionales en comparación con los estudios tradicionales.

Una de las artistas más importantes para la historiografía española es sin duda Luisa Roldán, la única escultora que llegó a ser nombrada escultora de Cámara. La cantidad de estudios en torno a su figura es realmente destacable. Aunque no podemos reseñarlos todos, hay que destacar las biografías realizadas por María García Olloqui en $1977^{91}$ y $2000^{92}$, o la obra dirigida por José Luis Romero Torres y Antonio

84 Ann Smith, T. (1997). Reconsiderando el papel de la mujer en la Real Academia de Bellas Artes de San Fernando. En W. Rincón García (dir.), La mujer en el arte español: Actas de las VIII Jornadas de Arte (pp. 279-288). Madrid: Alpuerto.

85 López Castán, Á. (1997). Las escuelas femeninas de encajes en el Madrid ilustrado. En W. Rincón García (dir.), La mujer en el arte español: Actas de las VIII Jornadas de Arte (pp. 265-270). Madrid: Alpuerto.

86 Castro Brunetto, C. J. (1997). La mujer en la creación artística del barroco canario. En W. Rincón García (dir.), La mujer en el arte español: Actas de las VIII Jornadas de Arte (pp. 271-278). Madrid: Alpuerto.

87 López Palomares, E. (1995). Mujeres en la Real Academia de Bellas Artes Artes de San Carlos: 1768-1849. Asparkia: Investigació Feminista, 5, 37-46.

88 Aranda Bernal, A. (2007). Ser mujer y artista en la Edad Moderna. En J.L. Romero Torres (dir.) y A. Torrejón Díaz (dir.), Roldana (pp. 33-54). Madrid: Consejería de Cultura. Más adelante hablaremos de la obra donde se encuentra este capítulo.

89 Ibídem, 41.

90 Como es evidente, dentro de estos estudios también puede hablarse de aspectos generales que afectan a todas las mujeres, sin embargo, son las mujeres concretas las protagonistas de ellos. Por ejemplo, en el capítulo escrito por Ana Aranda Bernal que hemos mencionado anteriormente, nos encontramos ante un contenido aplicable a todas las mujeres, pero la principal protagonista de la obra en la que se encuentra es una mujer concreta, Luisa Roldán.

91 García Olloqui, M. V. (1977). La Roldana. Sevilla: Diputación Provincial de Sevilla.

92 García Olloqui, M. V. (2000). Luisa Roldán: La Roldana, nueva biografía. Sevilla: Guadalquivir. 
Torrejón Díaz que hemos mencionado anteriormente, Roldana ${ }^{93}$. También hay varios artículos sobre obras concretas de la escultora, como «Un nuevo Ecce Homo de La Roldana y el mensaje teológico de esta iconografía en la escultura barroca española» de Fernando Llamazares Rodrígue ${ }^{94} \mathrm{o}$ «Dos cabezas cortadas atribuibles a Luisa Roldán en la Hispanic Society of America» de Alfonso Pleguezuelo Hernández ${ }^{95}$.

Hay más mujeres de las que podemos encontrar estudios específicos, como la pintora Josefa de Ayala ${ }^{96}$, Estefanía Gaurre del Canal (conocida como Estefanía de la Encarnación cuando tomó los hábitos) ${ }^{97}$, la grabadora Ana Heylan ${ }^{98}$, Cecilia Sobrino (conocida como Cecilia del Nacimiento cuando ingresó en religión) ${ }^{99}$ y Teresa Sarmiento de la Cerda, también pintora ${ }^{100}$. Por desgracia, todas ellas son excepciones. Muy pocas mujeres cuentan con estudios dedicados a ellas, y casi ninguna cuenta con tantos como Luisa Roldán.

- En segundo lugar, dentro de este tercer periodo en el que hemos dividido la historiografía española, podemos encontrar todos aquellos estudios que abordan a las mujeres artistas de una manera indirecta. Son obras centradas en otros temas, pero en algún momento se trata a las mujeres artistas en ellos, ya sea de manera general o específica como hemos visto antes.

Cuando lo hacen de forma general, suelen ser obras sobre la época. Nos hablan de la sociedad o política de ese momento y, en cierto punto también se hace mención a las mujeres artistas y como encajaba su figura en ellos, aunque sea muy brevemente. Podemos encontrar muchos tipos de obras diferentes. Desde diccionarios de artistas donde aparecen algunas referenciadas hasta estudios de la época donde también se mencionan artistas de ese momento. Por ejemplo, en el libro Introducción al arte barroco: el gran teatro del mundo, de Beatriz Blasco Esquivias ${ }^{101}$, encontramos un

93 Romero Torres, J.L. (dir.) y Torrejón Díaz, A. (dir) (2007). Roldana. Madrid: Consejería de Cultura.

94 Llamazares Rodríguez, F. (2009-2010). Un nuevo Ecce Homo de La Roldana y el mensaje teológico de esta iconografía en la escultura barroca española. Boletín de Arte, 30-31, 87-95.

95 Pleguezuelo Hernández, A. (2016). Dos cabezas cortadas atribuibles a Luisa Roldán en la Hispanic Society of America. Archivo Español de Arte, 353, 29-42.

96 Sobre esta mujer ya hemos mencionado algunas obras y estudios en las páginas anteriores. Aun así, podríamos destacar Sullivan, E. J. (1981). Obras de Josefa de Ayala, pintora ibérica. Archivo Español de Arte, 213, 386387; Sobral, L. de M. (1984). Un nuevo cuadro de Josefa de Ayala (o de Obidos). Archivo Español de Arte, 228, 87-93.

97 Aunque resulta muy destacable su autobiografía, de la cual hemos hablado en las páginas anteriores, destacan los dos estudios de María Isabel Barbeito Carneiro, centrados en el periodo religioso de la artista. Barbeito Carneiro, M. I. (1987). Una madrileña polifacética en Santa Clara de Lerma: Estefanía de la Encarnación. Anales del Instituto de Estudios Madrileños, 24, 151-163; Barbeito Carneiro, M. I. (2007). En Él fueron transformadas. Via Spiritus: Revista de Historia da Espiritualidade e do Sentimento religioso, 14, 31-64.

98 Moreno Garrido, A. (1990). La iconografía de la Virgen Antigua en el grabado granadino del siglo XVII: Una plancha inédita de Ana Heylan. Cuadernos de arte en la Universidad de Granada, 21, 205-210.

99 Ya hemos mencionado el estudio de Blanca Alonso-Cortés donde se hablaba de la madre y de la hija, pero podemos encontrar otros como el de Luis Javier Fernández Frontela, centrado específicamente en Cecilia Sobrino. Fernández Frontela, L. J. (2013). Cecilia del Nacimiento, Monja Carmelita Descalza y escritora. Revista de Espiritualidad, 287, 159-192.

100 García López, D. (2011). La IX duquesa Béjar doña Teresa Sarmiento y el monasterio de franciscanas de la Purísima Concepción de Caballero de Gracia. Las mujeres y la práctica de la pintura en la España del siglo XVII. En F. J. Campos y Fernández de Sevilla (coord.), La clausura femenina en el Mundo Hispánico: una fidelidad secular (pp. 865-881). Madrid: Real Centro Universitario Escorial; Este mismo autor tiene un capítulo en la obra Estudios de platería: San Eloy 2006, coordinado por Jesús Rivas Carmona, titulado «Joyería y platería en la Casa de Béjar, 1660-1677: Las compras de la duquesa doña Teresa Sarmiento de la Cerda, IX duquesa de Béjar». Sin embargo, en este capítulo no se habla de las facetas artísticas de dicha mujer, solo de su colección.

101 Blasco Esquivias, B. (2015). Introducción al arte barroco: el gran teatro del mundo. Madrid: Cátedra. 
capítulo, «Otro "género" de pintura. Mujeres pintoras (y una escultora)», completamente dedicado a las mujeres artistas de la Edad Moderna, tanto de Europa en general como de España en particular ${ }^{102}$.

Si hablan de mujeres concretas, generalmente nos encontramos ante obras dedicadas a un artista masculino que tenía algún tipo de vinculación con dicha mujer. Eran hermanas, hijas o mujeres de este y el autor las menciona en referencia a él. En las páginas anteriores hemos visto monografías protagonizadas por mujeres concretas, como Luisa Roldán o Josefa de Ayala, sin embargo, son excepciones. La mayoría de los estudios sobre mujeres concretas se hallan introducidos dentro de una investigación protagonizada por un artista masculino. Por ejemplo, no tenemos estudios específicos de Dorotea y Margarita Macip, sin embargo, aparecen mencionadas en muchas obras dedicadas a su padre ${ }^{103}$. Algo parecido encontramos con Isabel Sánchez Coello, que aparece tratada en varios trabajos sobre su padre, Alonso Sánchez Coello ${ }^{104}$. Lo mismo ocurre con las hijas de Pedro de Mena, Andrea y Claudia de Mena $^{105}$. Curioso es el caso de María y Francisca Roldán, las hermanas de Luisa Roldán, ya que estas mujeres casi siempre suelen aparecer mencionadas en obras dedicadas a su hermana (recordemos que Luisa es una de las pocas mujeres artistas que si es protagonista dentro de la historiografía española $)^{106}$. A María Eugenia de Beer la encontramos en algunos libros sobre el grabado de esta época ${ }^{107}$. En muchas biografías dedicadas a Juan de Valdés Leal encontramos referencias a su mujer, Isabel Ca-

102 Ibidem, 114-120.

103 Ya hemos visto antes algunas obras sobre mujeres artistas donde estas hermanas aparecen mencionadas, sin embargo, también son tratadas en diferentes estudios sobre su padre. Podríamos destacar Dotor y Municio, A. (1953). Juan de Juanes: una familia de pintores. Gerona: Dalmáu Carles Pla; Albi, J. (1979). Joan de Joanes y su círculo artístico. Valencia: Institución Alfonso el Magnánimo; Puig Sanchis, I. (2015). El pintor Joan de Joanes y su entorno familiar: los Macip a través de las fuentes literarias y la documentación de archivo. Lleida: Universitat de Lleida.

104 Aunque encontramos varias obras donde aparece tratada esta mujer, como la ponencia de Stephanie BreuerHermann o el estudio sobre retratos en miniatura de Julia de la Torre Fazio, destaca el capítulo que le dedica María Kusche, «Isabel Sánchez Coello Reynalte», en su obra Retratos y Retratadores: Alonso Sánchez Coello y sus competidores Sofonisba Anguissola, Jorge de la Rúa y Rolán Moys (pp. 384-385), donde también habla de Sofonisba Anguissola. Aunque esta mujer artista no era española, estuvo varios años en nuestro país y la polémica sobre las obras que pudo realizar durante su estancia ha sido estudiada en numerosas ocasiones por la propia María Kusche. Breuer-Hermann, S. (1990). Alonso Sánchez Coello. Vida y obra. En J. M. Serrera (coord.), Alonso Sánchez Coello y el retrato en la Corte de Felipe II (pp.13-35). Madrid: Museo del Prado; Kusche, M. (2003). Retratos y Retratadores: Alonso Sánchez Coello y sus competidores Sofonisba Anguissola, Jorge de la Rúa y Rolán Moys. Madrid: Fundación de Apoyo al Arte Hispánico; Torre Fazio, J. de la (2009). El retrato en miniatura bajo los reinados de Felipe II y Felipe III. Málaga: Universidad de Málaga.

105 En el Simposio Nacional celebrado en Málaga en el año 1989 podemos encontrar algunas ponencias donde aparecen. Sin embargo, es en la obra de Gila Medina Lázaro donde se pone especial interés en las hijas del escultor. Morales Folguera, J. M. (coord.) (1990). Pedro de Mena y su época. Sevilla: Junta de Andalucía, Dirección General de Bienes Culturales; Lázaro, G. M. (2007). Pedro de Mena escultor: 1628-1688. Madrid: El Autor, Arco Libros.

106 Son muchas las obras de Luisa Roldán donde aparecen mencionadas sus hermanas. Podríamos destacar Torrejón Díaz, A. (2007). El entorno familiar y artístico de La Roldana: el taller de Pedro Roldán. En J.L. Romero Torres (dir.) y A. Torrejón Díaz (dir.), Roldana (pp. 53-78). Madrid: Consejería de Cultura; En el caso de Francisca, esta también aparece mencionada y tratada en algunos estudios sobre su hijo, Pedro Duque Cornejo, como ocurre en el artículo de José Cesáreo López Plasencia, donde se dice, entre otras cosas, que Pedro Duque aprendió a estofar y policromar de su madre. Cesáreo López Plasencia, J. (2001). Escultura sevillana en Canarias: Una nueva obra de Pedro Duque Cornejo en Tenerife. Anuario de estudios atlánticos, 47, 161-178.

107 Podríamos destacar la obra de Antonio Gallego, donde se habla extensamente de su producción. Gallego, A. (1979). Historia del grabado en España. Madrid: Cátedra. 
rrasquilla, y sus dos hijas, Luisa y María Valdés, que también eran artistas ${ }^{108}$. Como han dicho algunos autores, cuando estudiamos mujeres artistas, muchas veces nos damos cuenta de que en realidad estamos estudiando a sus familiares masculinos.

Al margen de las dos categorías en las que hemos dividido la historiografía española de este tercer periodo, también podemos mencionar, aunque sea muy brevemente, las traducciones que se realizan de algunas obras extranjeras sobre el tema en nuestro país. Lo primero que deberíamos destacar es que, por desgracia, en algunas ocasiones, estas traducciones llegan varios años después de la publicación original, lo que provoca un desfase en algunos temas entre la historiografía española y la que se realiza en otros países. Por ejemplo, el famoso artículo de Linda Nochlin, «Why Have There Been No Great Women Artists?», publicado originalmente en 1971, no llegó traducido a España hasta el catálogo de la exposición Amazonas del arte nuevo, celebrada en 2008 (más de 30 años después) ${ }^{109}$. Como es evidente, este retraso tan notable no se produce en todos los casos. Women, Art and Society de Whitney Chadwick fue publicada en 1990 y tan solo dos años después fue traducida al español por la editorial Destino ${ }^{110}$. También podríamos destacar la obra A History of Own: Women in Europe from Prehistory to the Present, escrita por Bonnie S. Anderson y Judith P. Zinsser en 1988. Cuando se tradujo a nuestro idioma en 1991 bajo el título de Historia de las mujeres: una historia propia ${ }^{111}$, se le añadió un anexo titulado «Historias de las mujeres en España», dirigido por Gloria Nielfa Cristóbal, completamente centrado en nuestro país ${ }^{112}$. Esta obra abarca la historia de las mujeres en general, pero también se menciona a las artistas.

Podríamos concluir diciendo que, hasta los últimos años, la historiografía española ha pasado muy por encima de las mujeres artistas en la España de la Edad Moderna. No ha sido hasta las últimas décadas cuando se han empezado a impulsar los estudios sobre ellas y, poco a poco, han dejado de estar ignoradas. Es cierto que algunas mujeres de fama han contado con más estudios que otras a lo largo de la historiografía, sin embargo, son excepciones en comparación con la mayoría, que tras su muerte, eran prácticamente olvidadas por muchos autores posteriores.

Este creciente interés en nuestro país durante los últimos años también ha desencadenado en la creación de varios organismos y centros de investigación oficiales

108 Ninguna de estas mujeres cuentan con un estudio centrado en ellas, sin embargo, aparecen mencionadas de algún modo en casi todos los libros que podemos encontrar sobre Juan de Valdés Leal. Hemos mencionado antes el estudio de Gestoso y Pérez antes, pero hay muchos más. Algunos autores, como Enrique Pareja López, relacionan a esta Luisa con Luisa Morales, una destacable grabadora de la época. Gestoso y Pérez, op. cit., 1917; Valdivieso, E. (1988). Juan de Valdés Leal. Sevilla: Guadalquivir; Pareja López, E. (com.) (2001). Juan de Valdés Leal (1622-1690). Córdoba: Publicaciones de la Obra Social y Cultural CajaSur.

109 Nochlin, L. (2008). ¿Por qué no ha habido grandes mujeres artistas? (trad. Margarita Hernando de Larramendi). En J. Casamartina i Parassols, J. (com.) y Jiménez Burillo, P. (com.), Amazonas del arte nuevo (pp. 283-289). Madrid: Fundación Mapfre (Original en inglés, 1971).

110 Chadwick, W. (1992). Mujer, arte y sociedad (trad. M. Barberán). Barcelona: Destino (Original en inglés, 1990).

111 Anderson, B. S. y Zinsser, J. P. (1991). Historia de las mujeres: una historia propia (trad. Teresa Camprodón y Beatriz Villacañas), Vol. I y II. Barcelona: Crítica (Original en inglés, 1988).

112 Este anexo fue realizado por el Instituto de Investigación Feministas de la Universidad Complutense de Madrid. Aparte de Gloria Nielfa Cristóbal (Universidad Complutense de Madrid), que también se encarga de coordinar el anexo, podemos encontrar otras autoras como Cándida Martínez López (Universidad de Granada), MaríaMilagros Rivera (Universidad de Barcelona), Mariló Vigil Medina (Universidad Complutense de Madrid), Carmen Sarasúa García (Universidad Complutense de Madrid), Gloria Nielfa Cristóbal (Universidad Complutense de Madrid), Pilar Domínguez Prats (Universidad Complutense de Madrid), Ma Carmen García-Nieto París (Universidad Complutense de Madrid) y Concha Fagoaga (Universidad Complutense de Madrid). 
especializados en el tema, casi siempre vinculados a una universidad. Algunos de los centros que podemos destacar son el Centre de Recerca de Dones (DOUDA) en Barcelona $^{113}$, el Instituto Universitario de Investigación de Estudios de las Mujeres y de Género en la ciudad de Granada ${ }^{114}$, el Instituto Universitario de Estudios de las Mujeres en Tenerife ${ }^{115}$, el Instituto Universitario de Estudios y de Género "Purificación Escribano" de Castellón ${ }^{116}$, el Institut Universitari d'Estudis de la Dona en Valencia $^{117}$, el Instituto de Estudios de la Mujer en Madrid ${ }^{118}$ o, también de Madrid, el Instituto de Investigaciones Feministas ${ }^{119}$, entre otros.

Lo mismo ocurre con algunas revistas especializadas, como DUODA: Revista d'estudis feministes $^{120}$, Asparkia: Investigació feminista ${ }^{121}$, Arenal: Revista de historia de las mujeres ${ }^{122}$ e Investigaciones Feministas: papeles de estudios de mujeres, feministas y de género ${ }^{123}$, todas ellas vinculadas con alguno de los centros de investigación que hemos mencionado antes.

También se han llevado a cabo varios proyectos de investigación donde se ha profundizado en diversos aspectos de este tema. Podríamos destacar "El mecenazgo de la mujer y su papel en el desarrollo del arte y la arquitectura en la Plena Edad Media" (European Research Council Starting Grant no. 263036, 2010-2015) 124 "'La arquitectura en Andalucía desde una perspectiva de género: estudio de casos, prácticas y realidades construidas" (Proyecto de Excelencia HUM 5709, 2011-2015) ${ }^{125}$, "Lecturas de la Historia del Arte Contemporáneo desde la perspectiva de género" (HAR2011-22541, 2011-2016) ${ }^{126}$ y "Femenino Singular. Las mujeres y las artes en la corte española en la Edad Moderna (reinas, nobles, artistas y empresarias)" (Proyecto de Investigación $\mathrm{I}+\mathrm{D}+\mathrm{i}$ HAR2015-65166-P, MINECO/FEDER) ${ }^{127}$. La realización de estos proyectos ha culminado en la organización de múltiples seminarios y la publicación de varios estudios que se pueden consultar en sus respectivas páginas web ${ }^{128}$.

113 Centro de investigación fundado en 1982 y vinculado a la Universitat de Barcelona. Desde 1988 ofrece el Máster Estudios de la libertad femenina y, a partir del 2000, el Máster online Estudios de la diferencia sexual.

114 Su origen se remonta al Seminario de Estudios de la Mujer, que surge en el año académico 1984-85. Varios años después, en el curso 1995-96, el Seminario se convierte en el Instituto de Investigación, directamente vinculado con la Universidad de Granada. Desde el año 2015 coordina el programa oficial de Doctorado de Estudios de las Mujeres, Discursos y Prácticas de Género.

115 Vinculado a la Universidad de La Laguna, este Instituto se originó en 2007 a partir del antiguo Centro de Estudios de la Mujer, creado en 1995.

116 Aunque se funda en 2009, su origen se encuentra vinculado al de la propia Universitat Jaume I de Castellón, en 1991, con el Seminario de Investigación Feminista.

117 Instituto fundado en 1991 y relacionado con la Universitat de València. Su origen está en el Seminari Interdisciplinar d'Investigació Feminista de 1986.

118 Organismo de investigación fundado en 1993 en la Universidad Autónoma de Madrid.

119 Fue creado en el curso académico 1988-89 y está directamente relacionado con la Universidad Complutense de Madrid.

120 Publicada por la Universitat de Barcelona y el Centre de Recerca de Dones a partir de 1990.

121 Publicada por la Universitat Jaume I y el Instituto Universitario de Estudios y de Género "Purificación Escribano" a partir de 1993.

122 Publicada por la Universidad de Granada y el Instituto de Estudios de la Mujer a partir de 1994.

123 Publicada por la Universidad Complutense de Madrid a partir de 2009.

124 Página web: http: //www.proyectos.cchs.csic.es/womenasmakers/ [Consulta: 19 de febrero de 2018]

125 Página web: http: //www.ugr.es/ genarq/index.htm [Consulta: 19 de febrero de 2018]

126 Página web: http: //www.uma.es/departamento-de-historia-del-arte/info/106430/lecturas-de-la-historia-delarte-contemporaneo-desde-la-perspectiva-de-genero-lhcpg/ [Consulta: 19 de febrero de 2018]

127 Página web: http: //www.ucm.es/femenino_singular/ [Consulta: 19 de febrero de 2018]

${ }_{128}$ El más reciente ha sido el Seminario Internacional: Las mujeres y las artes en la corte española de la Edad Moderna, organizado por el Proyecto de Investigación I+D+i Femenino Singular los días 20, 21 y 22 de septiembre 
Los estudios sobre género y mujeres artistas se están introduciendo poco a poco en el ámbito universitario con la inclusión de algunas asignaturas y cursos completamente dedicados al tema. María Elena Díez Jorge fue pionera al impartir el curso de posgrado Ciudad, arquitectura y género en el Máster oficial del Departamento de Historia del Arte de la Universidad de Granada desde 2002 hasta 2016. La Universidad de Málaga imparte el Máster en Igualdad y Género desde el año académico 2010 / 2011. En el curso académico 2017/2018, la Universidad Autónoma de Madrid ha incluido en el Grado de Historia del Arte la asignatura optativa Arte y Género, impartida por María Cruz de Carlos Varona.

Aunque en el extranjero encontramos exposiciones de mujeres artistas en los museos desde la década de los $70^{129}$, en España no ha sido hasta el año 2016 cuando el Museo del Prado ha presentado una exposición protagonizada por una mujer artista: El arte de Clara Peeters, comisionada por Alejandro Vergara.

Como hemos dicho al principio, en este artículo no hemos pretendido hablar de todos los estudios en los que aparece alguna mujer artista mencionada. Hay muchos más de los que puede parecer a simple vista y sería imposible abarcarlos todos en estas páginas. Hemos intentado mencionar aquellos de especial relevancia para cada tipo de bibliografía que hemos tratado en cada momento (estudios de género, obras sobre mujeres artistas tratadas de forma directa o indirecta, etc.). Por ejemplo, podemos encontrar más estudios de artistas masculinos en los que también se menciona alguna mujer artista que tenía una vinculación con él. Sin embargo, en algunos casos se trata de menciones que apenas ocupan dos líneas. En las páginas anteriores hemos puesto ejemplos de aquellos casos más destacables.

Todavía nos siguen faltando estudios que analicen y profundicen en la figura de las mujeres artistas en España en la Edad Moderna de una forma general. La mayor parte de la información que se tiene se encuentra muy dispersa y, cuando profundizamos un poco en el tema, descubrimos que muchas de las cosas que se dan por sentadas en realidad se desconocen. A estas alturas, ya ha quedado más que demostrado que la participación de la mujer en el mundo del arte no era algo anecdótico, por ello debemos intentar recuperar esta parte de nuestro discurso artístico que todavía se desconoce en su mayor parte, y casi siempre se presenta como algo extraordinario, para así poder empezar a comprender la historia del arte con todos sus participantes. No se trata de hacer una historia del arte de hombres o una historia del arte de mujeres, sino una historia del arte donde ambos participantes estén integrados por igual y resulte completa en lo máximo posible.

de 2017. El programa está disponible en su página web: http: //www.ucm.es/femenino_singular/noticias/21060 [Consulta: 19 de febrero de 2018]

129 Women Artists: 1550-1950, celebrada en 1976 en el Museo de Arte del Condado de Los Ángeles y comisionada por Linda Nochlin y Ann Sutherland Harris, fue la primera exposición dedicada completamente a mujeres artistas. 